\title{
Research on Object Detection based on Mathematical Morphology
}

\author{
S.QIAN ${ }^{1} \&$ G.R.WENG ${ }^{2}$ \\ ${ }^{1}$ School of Mechanical and Electric Engineering, Soochow University, Suzhou, Jiangsu, China \\ ${ }^{2}$ School of Mechanical and Electric Engineering, Soochow University, Suzhou, Jiangsu, China
}

KEYWORD: Object detection; Mathematical morphology; Image segmentation

ABSTRACT: An approach for object detection based on mathematical morphology is proposed in this paper. The features of objects are extracted through the analysis of images, and structuring elements with different shapes and sizes are selected according to the aim of experiments. After the image preprocessing, a gray image is obtained, which is propitious to the analysis of computer. Then the image is used by a threshold and a binary image is achieved. At length, the objects are detected through mathematical morphology. Experimental results show that the proposed approach to object detection is feasible with Mathematical Morphology. And it has a great effect.

\section{Introduction}

With the continuous progress of the digital image processing technology, the image processing technology is applied more and more widely. In modern war, it is significant to identify and capture the object accurately. Scientists paid more attention to object detection and recognition, which recently has become one focus in this field.

Object detection is the combination of image segmentation and recognition. Image segmentation is a technology that divides an image into several uniform regions and extracts the regions needed from complex scene. Image recognition is on the basic of features of the image. Feature selection and extraction are the process of analyzing the preprocessed data, discarding the dross and selecting the essence.

In order to improve the speed and accuracy of classification, it is necessary to select the most representative feature with proportion, rotation and shift invariability.[1] According to different objects, the complexities of the selected features are quite different. Although they can also be the graph with complex structures or complete image of the object, the features can be simple ones such as point, line and block. As a rule, choosing simple features is good for feature extraction of images but it is bad for feature correspondence and matching.

An approach for object detection based on mathematical morphology is proposed in this paper. Analyze the structuring characteristics of the image, and object detection of image will be finished by morphological method.

\section{Image preprocessing}

In practice, the original images usually are not of high quality due to the existence of errors and noise during data acquisition. For the sake of effective analysis and the recognition through computers, image preprocessing is usually chosen to be the first step of the operations, which includes gray-level transformation, filters and image enhancement.

The median filter is a canonical image processing operation, best known for its salt and pepper noise removal aptitude. It is also the foundation upon which more advanced image filters like unsharp marking, rank-order processing, and morphological operations are built.[2] The median filter is a operation that sorts the items around the objects according to the pixel and takes the median one to replace the original value. It can effectively suppress interference like isolated points and line segments. Since values which are quite different from surrounding items have a limited effect on the median filter, it will preserve edges and details of the image when filtering the impulse noise and pepper noise. Compared to some Linear smoothing filters with small size, it has better effects. And it's widely used in image processing. 

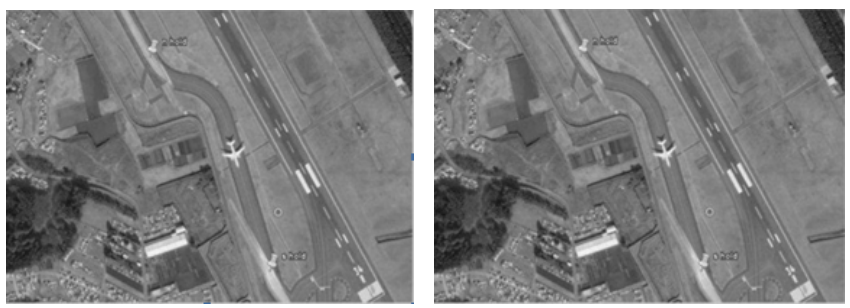

(a) grayscale image (b) image after image preprocessing

Figure 1. image preprocessing

\section{Threshold segmentation}

Because of its intuitive properties and simplicity of implementation, image thresholding is significant in applications of image segmentation. A thresholded image $\mathrm{g}(\mathrm{x}, \mathrm{y})$ is defined as

$$
g(x, y)= \begin{cases}1 & \text { if } f(x, y) \geq T \\ 0 & \text { if } f(x, y)<T\end{cases}
$$

Thus, pixels labeled 1(or any other convenient gray level) correspond to objects, whereas pixels labeled 0 (or any other gray level not assigned to objects) correspond to the background.[3] The key to image thresholding is to determine the threshold. An appropriate threshold can help partition an image accurately.

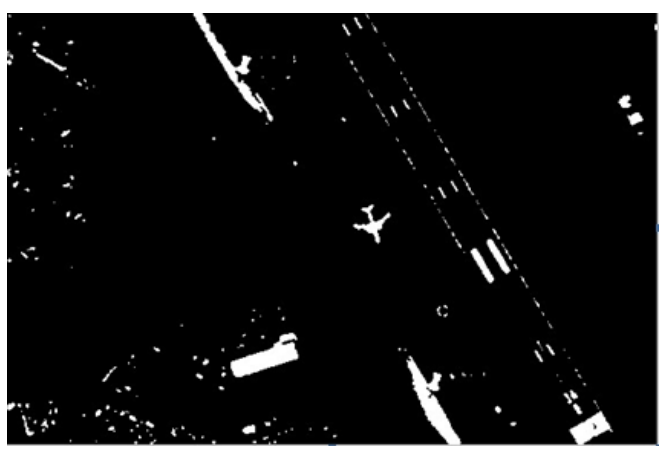

Figure 2. Thresholded image

\section{Morphology}

\section{structuring elements}

The basis of mathematical morphology is geometry. In order to analyze the image ,it is necessary to find probes with certain shapes to analyze the image. And the goal of recognition and image processing is achieved. In mathematical morphology, these probes are called structuring elements. In fact, through constantly moving the structuring element to apply to the objects, the features of image like brightness, color and shape are obtained and the geometric parameters of them are determined. [5]Structuring elements is an important concept of mathematical morphology, the selection of which has a great effect on the result of Morphological operation. Because of the flexibility, structuring elements of all shapes and sizes can be chosen according to the purpose of the experiment. Structuring elements of different shapes will have different effects on filters, so do the structuring elements of different sizes.

\section{Dilation and Erosion}

\section{Dilation}

Dilation is the process of expansion. In a binary image, dilation is an operation that "grow" or "thickens" objects. The extent of this thickening and lengthening is controlled by a structuring element. In math, dilation is defined in terms of set operation. The dilation of $\mathrm{A}$ by $\mathrm{B}$, denoted $\mathrm{A} \oplus \mathrm{B}$, is defined as[3] 
Dilation is an operation that increase the object size and narrows holes. Furthermore, it can fill small holes and narrow gulfs in objects. [4]

\section{Erosion}

Erosion is an operation that "shrinks" or "thins" objects in a binary image. Erosion has a great effect on small noise. As a dilation, the manner and extent of shrinking is controlled by a structuring elements.

Mathematically, erosion is also defined in terms of set operation. The erosion of A by B, denoted $\mathrm{A} \Theta \mathrm{B}$, is defined as[3]

$\mathrm{A} \Theta \mathrm{B}=\left\{\mathrm{z} \mid(\mathrm{B})_{z} \cap \mathrm{A}^{c}=\varnothing\right\}$

On the contrary to dilation, Erosion is an operation that increase holes and narrows the object size. Erosion is used to simplify the structure of an object-objects or their parts with width equal to one will disappear. It might thus decompose complicated objects into several simpler ones.[4]

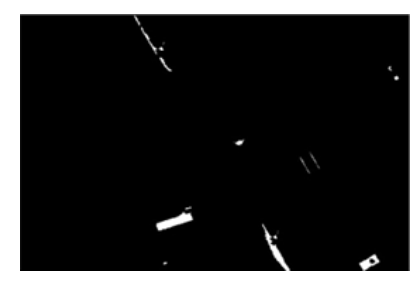

(a)

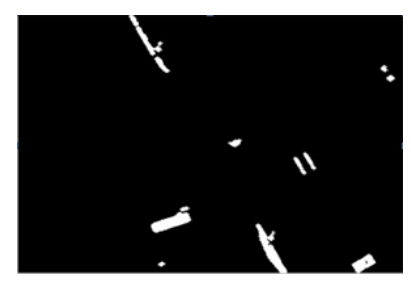

(b)

Figure 3. (a) shows the erosion (b) shows the dilation

\section{Morphological Reconstruction}

Reconstruction is a very important morphological transformation. In this operation, two images (marker f, mask g) and a structuring element are necessary. The structuring element used defines connectivity. But on condition that marker f must be a subset of g, that is $f \subseteq g$. The reconstruction of $\mathrm{g}$ from $\mathrm{f}$, denoted as $R_{g}(f)$, is defined by the following procedure:

1. Initialize $h_{1}$ to be the marker image $f$.

2.Creat the structuring element: $B=$ ones(3).

3.Repeat

$h_{k+1}=\left(h_{k} \oplus B\right) \cap g$

until $h_{k+1}=h_{k}$ [3]

Morphological reconstruction has a wide spectrum of practical application, such as filling holes, clearing border objects. Figure 4 shows the image that is processed with reconstruction. From the image, it is easy to find the object.

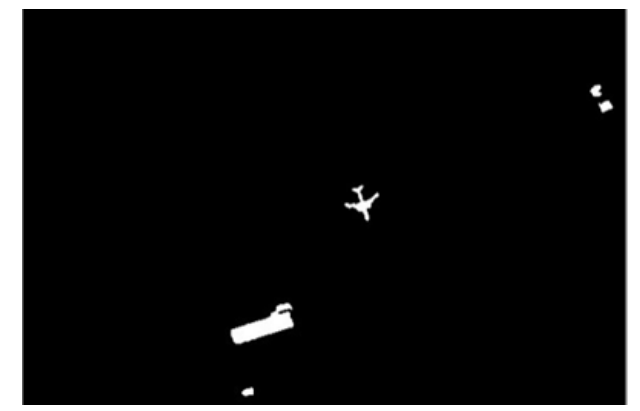

Figure 4. Image after reconstruction 


\section{Area opening}

Area morphology is based on area opening and closing. Structuring elements are not used in area opening and closing, which differs from traditional morphological filters. Therefore, these two operations on the image will not change the objects. Light objects which are smaller than the threshold are removed through area opening. Similar to area opening, dark objects which are smaller than the threshold are removed through area closing. As a smoothing filter, area opening has functions of smoothing the edge of image and removing small noise. The method has the advantages of simple algorithm, high accuracy. And it has been widely used in practice.

Area opening has a great effect on removing pepper noise. As Figure 5 shows, the objects are obtained by adjusting the threshold of area opening. With the operation of subtraction, the effect is better.

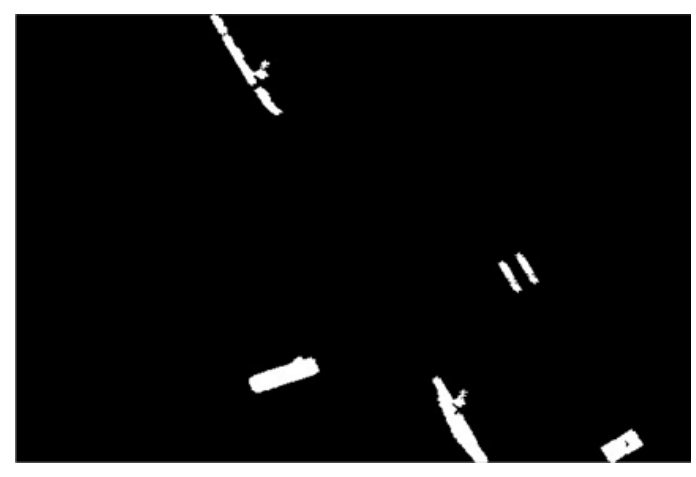

Figure 5. Area opening and Subtraction

\section{Labeling connected component}

The term connected component was defined in terms of a path, and the definition of a path in turn depends on adjacency. This implies that the nature of a connected component depends on which form of on adjacency we choose, with 4-and 8-adjacency being the most common.

Signature is 1-D functional representation of a boundary. Signature could be generated in various ways. One is to plot the distance from an inter point to the boundary as a function of angle. And this is a simplest and widely used way in digital image processing. Its basic idea is to reduce the boundary representation to a 1-D function, which is easier to describe.

\section{Fourier Descriptors}

Starting at an arbitrary point on a plane, coordinate pairs are encountered in the counterclockwise direction. These coordinates can be expressed in the form $x(k)=x_{k}$ and $y(k)=y_{k}$. And the boundary can be represented as the follow sequence of coordinate

$$
s(k)=[x(k), y(k)], k=0,1,2, \mathrm{~L}, K-1
$$

Furthermore, each coordinate pair can be treated as a complex number, and $\mathrm{s}(\mathrm{k})$ was defined as

$$
s(k)=x(k)+j y(k)
$$

the discrete Fourier transform(DFT) of $\mathrm{s}(\mathrm{k})$ is

$$
a(u)=\sum_{k=0}^{K-1} s(k) e^{-j 2 \pi u k / K}, \text { for } \mathrm{u}=0,1,2 \ldots, \mathrm{K}-1
$$

The inverse Fourier transform of $\mathrm{s}(\mathrm{k}) .[3]$

$$
s(k)=\frac{1}{K} \sum_{u=0}^{K-1} a(u) e^{j 2 \pi u k / K}
$$




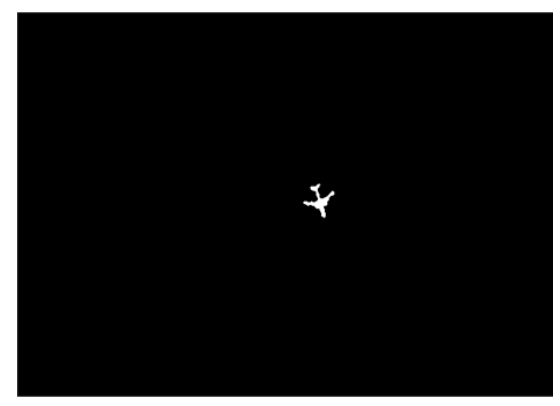

Figure 6. Object image: center (315.37,183.401), Area: 289 Num :1

\section{Experimental Results}

Compared with the original image Figure 7(a), Figure 7(b) shows that the object is extracted. The options of structuring elements and threshold are the main factors which influence the experimental results. Morphological transformation usually carries out the operation with the help of symmetrical structuring elements. When the structuring elements are not symmetry, the result of the operation will generally produce offsets. When the shapes of structural elements are determined, it is crucial to adjust the size of the structuring element. If the size is too small, fracture edge will not be connected through the operation of closing and large adhesion will not be removed through the operation of opening. On the contrary, if the size of structuring element is too bigger, the objects will interfere with each other through the operation of closing and false fracture will happen through the operation of opening. If the threshold is small, foreground will be mistaken for objects. If the threshold is too big, the foreground objects won't be complete. Therefore, it is significant to choose the appropriate threshold.

Choosing other images does the experiments with this algorithm, the effect is also good. And this demonstrated the feasibility of this method.
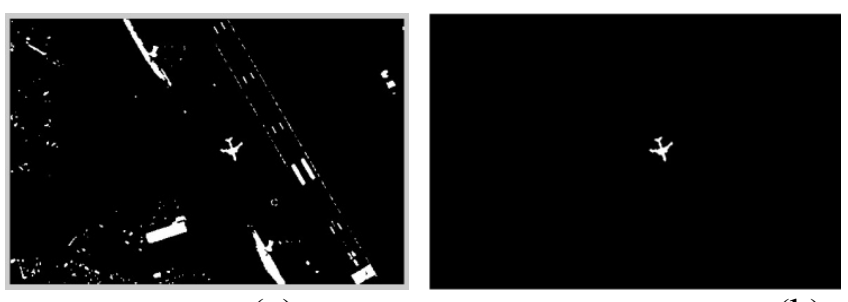

(a)

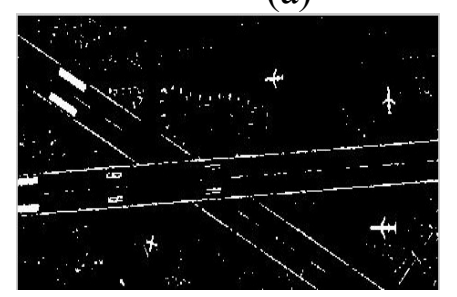

(b)

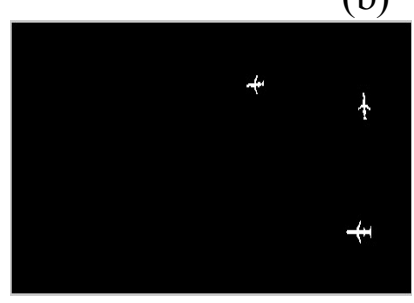

(c)

(d)

Figure 7. Comparison of detection results:

(a),(c) thresholded image ical method

(b),(d)results of morpholog-

\section{Conclusion}

An approach for object detection based on mathematical morphology is proposed in this paper. First, the image is using a threshold and a binary image is achieved. Then extracting the features of objects and choosing the appropriate structuring element. Finally, the objects are detected through morphology. The algorithm is influenced by the surrounding environment of objects. What's more. Applied to the image with complicated background, the result does not look good. And the algorithm needs to be further improved. 


\section{Acknowledgment}

This work is supported by the National Nature Science Foundation of China [grant number 61473201].

\section{References}

[1] Q.R.Zhang, Qiaorong, Z., Li, J., Huimin, X., \& Guochang, G. 2011. Image segmentation based on visual saliency. Journal of Image and Graphics, 16(5), 767-772.

[2] Maragos, P., \& Schafer, R. W. 1987. Morphological filters--Part II: Their relations to median, order-statistic, and stack filters. Acoustics, Speech and Signal Processing, IEEE Transactions on, 35(8), 1170-1184.

[3] Gonzalez, R. C., Woods, R. E., \& Eddins, S. L. 2004. Digital image processing using MATLAB. Noida :Pearson Education India.

[4] Sonka, M., Hlavac, V., \& Boyle, R. 2014. Image processing, analysis, and machine vision. Boston: Cengage Learning..

[5] Putera, S. I., \& Ibrahim, Z. 2010, June. Printed circuit board defect detection using mathematical morphology and MATLAB image processing tools. In Education Technology and Computer (ICETC), 2010 2nd International Conference on (Vol. 5, pp. V5-359). IEEE. 